\title{
LA COMUNICACIÓN EN LA IGLESIA LATINOAMERICANA'
}

\section{COMMUNICATION IN THE LATIN AMERICAN CHURCH}

\author{
Óscar Julián Cuesta Moreno²
}

\begin{abstract}
RESUMEN
El artículo tiene el propósito de recopilar las preocupaciones de la iglesia latinoamericana sobre los medios de comunicación. Para ello, recupera lo dicho por las autoridades eclesiásticas del continente en los documentos de las Conferencias del Episcopado Latinoamericano. Este proceso se hace de manera cronológica y desde un punto de vista laico. En la última parte, presenta unas reflexiones generales sobre las conferencias y su preocupación por los medios.
\end{abstract}

Palabras clave: Comunicación, conferencias, episcopado, iglesia latinoamericana, medios de comunicación.

\section{ABSTRACT}

This paper aims to gather the concerns of the Latin American Church on the media. To do so, it recovers what it was said by the ecclesiastical authorities of the continent in the documents of Latin American Episcopate Conferences. This process is done in chronological order and from a secular point of view. In the last part it presents some general reflections on the conference and media concern.

Key words: Communication, conferences, episcopate, Latin American Church, media.

Recibido: 16 de noviembre de 2011 Aceptado: 15 de diciembre de 2011

\section{INTRODUCCIÓN}

El vínculo entre la Iglesia Católica y la comunicación es íntimo desde el origen mismo del cristianismo. Así lo hace pensar, por ejemplo, el perfil de comunicadores evidente en los apóstoles, quienes inspirados por el Espíritu Santo difundieron su fe más allá de las fronteras de Palestina y del pueblo judío. Su mensaje, manifiesto también en la escritura, Ilegó hasta la capital del Imperio Romano.

Hoy, fácilmente vemos el mensaje de Cristo difundido en diferentes canales, pues la Iglesia y sus feligreses utilizan la prensa, la radio, la televisión y los medios virtuales en sus propósitos. Además, su divulgación va de lo local a lo global: la televisación de una misa en un pueblo de Colombia por un canal comunitario, o la transmisión satelital de una homilía del Papa en Semana Santa.

1 Este artículo nace de una investigación realizada en la escritura del Proyecto Educativo del Programa (PEP) de Comunicación Social de la Universidad Santo Tomás de Bogotá.

2 Óscar Julián Cuesta Moreno: Comunicador Social de la Universidad Santo Tomás, Colombia. Magister en educación de la Universidad Pedagógica Nacional. oscarcuesta@colombia.com. 
Se pueden citar varios autores que han trabajado el tema de la iglesia y la comunicación. Recientemente, Babin (2011) analiza cómo evangelizar en esta dinámica de la sociedad conectada. Por su parte, Raigón (2010) observa la estructura de la información en la iglesia católica. Décadas atrás, comenzando esta línea, Pascual (1976) ya abordaba el tema de los medios y su relación con la doctrina católica.

Este artículo pretende aportar a este marco, pero limita su alcance a dar cuenta de la comunicación en las cinco Conferencias del Episcopado Latinoamericano, para lo cual toma como referente los documentos finales de las discusiones de los monarcas de este continente.

Cercano a este propósito, y a manera de antecedentes, se encuentran las publicaciones de Spoletini (1977 y 1985). Igualmente, se puede citar al mismo Consejo Episcopal Latinoamericano (1988), que publicó un libro sobre la teoría y praxis de la iglesia en la comunicación social. Este artículo pretende ser novedoso, básicamente por tres razones: porque brinda una mirada laica a las citadas conferencias, pues al fin de cuentas, se puede ver la cercanía de ellas con la iglesia; en segundo lugar, porque da cuenta de documentos del episcopado latinoamericano que los anteriores no mencionan, como las conferencias de Santo Domingo y Aparecida, que fueron realizadas muchos años después; y en tercer lugar, porque procura observar, en décadas posteriores, las preocupaciones del episcopado latinoamericano frente a los medios.

\section{METODOLOGÍA}

Para dar cuenta de cómo se ha abordado la comunicación en las Conferencias del
Episcopado latinoamericano, se efectuó una investigación de corte cualitativo; específicamente se realizó un revisión documental de los cinco informes finales de las cinco Conferencias Generales, es decir, de los documentos de Puebla, Medellín, Santo Domingo, Aparecida y Río de Janeiro. En una segunda fase, para ampliar el marco de análisis, se revisaron otros documentos del Episcopado que abordan el tema de la comunicación de manera explícita.

Para realizar el análisis, la lectura y la revisión de los documentos, se estableció como categoría principal la comunicación y como subcategoría los medios de comunicación. En esa línea, se procuró seguir palabras claves como comunicación, medios, radio, televisión, cine y prensa. A lo largo del proceso, emergieron relaciones categoriales como comunicación-educación y comunicación-cultura, que enriquecieron los resultados y la consecuente discusión.

La estructura del texto no responde a estas fases de investigación, pues procura dar cuenta de cada conferencia de manera cronológica. Por lo tanto, empieza con la conferencia de Río de Janeiro y termina con la de Aparecida. En el siguiente ítem se presentan simultáneamente los resultados y su análisis (discusión) en un mismo apartado, para hacer observaciones a cada documento.

\section{RESULTADOS Y DISCUSIÓN}

En el contexto continental se puede ver el interés de la iglesia católica por reflexionar sobre la comunicación. En las cinco Conferencias Generales del Episcopado latinoamericano se tocó de una u otra manera, el tema comunicacional. Pertinente es, en ese orden de ideas, recoger algunos planteamientos formulados en esos encuentros episcopales. 
En la Primer Conferencia General del Episcopado latinoamericano, realizada en Río de Janeiro en agosto de 1955, se aborda la posibilidad de utilizar la radio y la prensa "para difundir e inculcar más eficazmente la palabra sagrada y las enseñanzas de la Iglesia" (Documento de Río de Janeiro, 1955, p. 3). El Episcopado reconoce la creciente importancia de los medios en la sociedad de mediados de siglo, en la cual la radio no había tenido una total masificación y la televisión era un medio que hasta ahora incursionaba.

En esta conferencia se invita para que el Episcopado de cada país organice un diario nacional. Igualmente, Ilama la atención para que los diarios en circulación sean más atractivos a los ojos de los lectores. Del mismo modo, invita a que se celebre anualmente una jornada de la prensa católica. Además, sugiere que se cree una confederación interamericana de diarios católicos.

Por otro lado, motiva el uso de la radio y reconoce las posibilidades que puede brindar un medio de estas características en países de gran extensión y con poco número de clérigos. Para ello, enfatiza en que es importante "dar normas concretas y oportunas, aún en el plano diocesano, sobre la organización y el empleo del apostolado radiofónico" (Documento de Río, 1955, p. 25).

De manera particular, en el punto 62 del documentoseaconsejaseguirpromoviendo escuelas de periodismo para sacerdotes y laicos. Más puntualmente, en el punto 68 recomienda "fervorosamente el estudio y la fiel observancia de las luminosas enseñanzas pontificias relativas al cine, la radio y la televisión" (Documento de Río, 1955, p. 25).
La Segunda Conferencia General del Episcopado latinoamericano, realizada en Medellín en noviembre de 1968, afirma que la Iglesia latinoamericana tiene conciencia de las cualidades técnicas de los medios de comunicación social y sus posibilidades en la educación social, la concientización en el cambio de las estructuras sociales y la vigencia de la justicia. En pocas palabras, considera que los medios de comunicación deben ser utilizados en la realización de su misión y en la transformación de las inequitativas naciones latinoamericanas. Concluye que hay que "despertar en los hombres y en los pueblos, principalmente con los medios de comunicación social, una viva conciencia de justicia, infundiéndoles un sentido dinámico de responsabilidad y solidaridad" (Documento de Medellín, 1968, p. 25).

Al abordar el tema de la juventud, la conferencia de Medellín reconoce que los jóvenes necesitan formar una personalidad humana y cristiana, que les permita tener criterios frente a la influencia de los medios de comunicación y sus procesos de masificación. A la par que reconoce las posibles influencias negativas de los medios, invita a indagar sobre los recursos que estos brindan en el desarrollo de una catequesis apropiada: “Es, pues, urgente una seria investigación sobre el efecto de los medios de comunicación social y una búsqueda de la forma más adecuada de dar una respuesta, utilizándolos en la tarea evangelizadora, como también una seria evaluación de las realizaciones actuales" (Documento de Medellín, 1968, p. 61).

El documento de Medellín afirma que la comunicación social es una de las principales dimensiones de la humanidad, dimensión que según la conferencia, tiende a aumentar su impacto por la influencia del satélite y los avances electrónicos. 
En ese orden, considera que los medios de comunicación social "forjan una nueva cultura, producto de la civilización audiovisual que, si por un lado tiende a masificar al hombre, por otro favorece su personalización. Esta nueva cultura por primera vez se pone al alcance de todos, alfabetizados o no, lo que no acontecía en la cultura tradicional que apenas favorecía a una minoría" (Documento de Medellín, 1968, p. 102).

La conferencia de 1968, que toca ampliamente el tema de los medios de comunicación, ve en ellos posibilidades de transformación, pues tienen la capacidad de concientizar a las personas. Sin embargo, advierte que "muchos de estos medios están vinculados a grupos económicos y políticos nacionales y extranjeros, interesados en mantener el «statu quo» social" (Documento de Medellín, 1968, p. 103).

En Medellín, el episcopado latinoamericano hacía hincapié en la importancia de que la pastoral formara a los nuevos seminaristas en la utilización de los medios de comunicación (puesto que, en sus palabras, recibe gozosa la ayuda de estos, ya que sin ella le es imposible llegar a todos los hombres. El episcopado también ve con buenos ojos las dinámicas de grupo y las relaciones humanas, aspecto este que hace un reconocimiento tácito a la comunicación interpersonal.

De alguna manera la conferencia empieza a reconocer la relación entre la comunicación y la cultura en este apartado: "Se debe estimular la producción de un material adaptado a las variadas culturas locales (por ejemplo, artículos de prensa, emisiones radiofónicas y televisivas) para que promueva los valores autóctonos y sea convenientemente recibido por los usuarios" (Documento de Medellín, 1968, p. 105). Finalmente, la conferencia de Medellín reconoce la celebración del Día Mundial de la Comunicación social como una oportunidad para sensibilizar a las personas sobre la trascendencia de la misma en la vida del hombre y de la sociedad.

Por su parte, la Tercera Conferencia General del Episcopado latinoamericano, realizada en Puebla en marzo de 1979, habla de los medios de comunicación como transmisores de cultura. Alerta sobre la manipulación que ejercen distintos poderes en los medios, centrando su atención en la publicidad, la cual considera que genera falsas expectativas y a veces vulnera la cultura latinoamericana. Se puede decir que esta conferencia hace una mirada antropológica de los medios, en la medida en que centra su atención reiteradamente en la cultura, sobre todo en el marco de una sociedad globalizada por los medios, que además, transmiten pautas y modelos culturales nuevos.

En Puebla, el Episcopado muestra una evidente preocupación por la influencia de los medios en la moral de las naciones latinoamericanas, considera que es una amenaza para la familia el influjo de estos en la idolatría al poder, la riqueza y el sexo, además de que "contribuyen a propagar el divorcio, la infidelidad conyugal y el aborto o la aceptación del amor libre y de las relaciones pre-matrimoniales"(Documento de Puebla, 1979, p. 100).

El documento de Puebla sigue relacionando íntimamente la comunicación con los medios y reconoce la importancia de aquella en estos términos: "La comunicación social incide en toda la vida del hombre y ejerce sobre él de manera consciente o subliminal, una influencia decisiva". Reconoce, en ese sentido "que los medios de comunicación social son factores de 
comunión y contribuyen a la integración latinoamericana así como a la expansión y democratización de la cultura; contribuyen también al esparcimiento de las gentes que viven especialmente fuera de los centros urbanos; aumentan las capacidades perceptivas por el estímulo visual-auditivo, de penetración sensorial" (Documento de Puebla, 1979, p. 169).

El Episcopado es autocrítico al afirmar que "no existe todavía en la Iglesia de América Latina una verdadera preocupación para formar al pueblo de Dios en la comunicación social" (Documento de Puebla, 1979, p. 171), sobre todo para capacitar a las personas para que tengan una actitud crítica frente al mensaje de los medios. De ahí que invite a permanecer atentos y hacer seguimiento a los profesionales de la comunicación, especialmente, los que cubren información religiosa.

De manera particular, en esta conferencia se hace un llamado al uso de lo que ella llama los Medios de Comunicación Grupal (MCG), que hoy podríamos denominar medios comunitarios, pues son menos costosos y posibilitan más la comunicación persona a persona. En ese orden de ideas, también convoca a que se use el lenguaje adecuado y cercano a la realidad de la comunidad.

En octubre de 1992, en Santo Domingo, la Cuarta Conferencia General del Episcopado latinoamericano continuó tocando el tema de la comunicación como un campo íntimamente ligado a los medios. Cita las siguientes palabras del Papa Pablo VI, refiriéndose a los medios de comunicación: "La Iglesia se sentiría culpable ante Dios si no empleara esos poderosos medios, que la inteligencia humana perfecciona cada vez más".
El Episcopado sigue haciendo hincapié en la importancia de los medios de comunicación en la formación de las personas y en la difusión de la evangelización y exhorta de manera particular a acercarse a la cultura urbana (Documento de Santo Domingo, 1992, p. 77).

De manera novedosa, en Santo Domingo se habla de la Nueva Evangelización, forma de inculcar el mensaje de la Iglesia teniendo en cuenta la diversidad cultural de los pueblos: "Urge aprender a hablar según la mentalidad y cultura de los oyentes, de acuerdo a sus formas de comunicación y a los medios que están en uso" (Documento de Santo Domingo, 1992, p. 20). Esta conferencia se preocupa por la formación de laicos para que sobresalgan en los medios de comunicación social y en otros campos.

De una manera especial, no evidente en las anteriores conferencias, el Episcopado latinoamericano habla de la comunicación alejándose de los medios:

Jesucristo nos da la vida para comunicarla a todos. Nuestra misión nos exige que, unidos a nuestros pueblos, estemos abiertos a recibir esta vida en plenitud, para comunicarla abundantemente a las Iglesias a nosotros encomendadas, y también más allá de nuestras fronteras (...)Al mismo tiempo nos duele ver cómo muchos de nuestros fieles no son capaces de comunicar a los demás la alegría de su fe. Aquí se ve una íntima relación del compromiso comunicativo, tanto del clérigo como del laico, con el mensaje del evangelio (Documento de Santo Domingo, 1992, pp. 48-50).

Al igual que en Puebla, se vuelve a tocar la crisis que vive la familia y expone que es importante capacitar dicha institución 
para el uso de la TV, la prensa y la radio. En esa misma línea reconoce el crecimiento de grandes emporios mediáticos en América latina, pero juzga que estos están imponiendo una cultura que estimula el hedonismo y el consumismo, atropellando la cultura y la identidad latinoamericanas.

Teniendo en cuenta lo anterior, apoya los esfuerzos que pretenden defender la identidad cultural de los pueblos frente a los medio e invita a articular la comunicación masiva con la comunitaria y grupal (por primera vez se habla de comunicación comunitaria en las conferencias).

La V Conferencia General del Episcopado latinoamericano, realizada en Aparecida en mayo de 2007, presenta un cambio significativo respecto de las anteriores, puesto que discute sobre la capacidad de interactuar en tiempo real en una red mundial. Esto significa que se pasa de una primera inquietud por la radio y la prensa, a una segunda por la televisión, una tercera por la influencia cultural de los medios y su publicidad, y aún, una cuarta por las nuevas tecnologías y la Internet.

En Aparecida la preocupación por la globalización cultural es mayor; incluso habla de una "nueva colonización cultural por la imposición de culturas artificiales, despreciando culturas locales y tendiendo a imponer una cultura homogenizada en todos los sectores" (Documento de Aparecida, 2007, p. 14). Esta nueva cultura se caracteriza por la auto-referencia del individuo y, consecuentemente, la indiferencia por el otro, lo que lleva a ver las relaciones humanas como objeto de consumo. Como caso particular, hace un llamado frente a la vulneración que hacen los medios de la dignidad de las mujeres, pues las trata como objetos sexuales $y$ fuente de lucro.
Por otro lado, observa que hay culturas que exigen reconocimiento y que han generado respuestas no solo a la globalización, de lo cual se había hablado antes de Aparecida, sino a los estereotipos de los medios de comunicación.

Para la conferencia, la globalización es un fenómeno complejo que exige una justa valoración que permita identificar sus consecuencias positivas y negativas. Sin embargo, lamenta que la cara más difundida de la globalización sea su dinámica económica, que prevalece sobre otras dimensiones de la vida humana, al centrar su acción en el mercado.

El Episcopado se preocupa por el uso que dan los jóvenes a la comunicación virtual. Del mismo modo, hace un llamado para que se encaminen esfuerzos para contrarrestar la cultura posmoderna que emiten los medios, porque debido a ellos, el individuo tiene una noción vaga del futuro y procura una vida efímera y sin grandes metas.

A diferencia de las anteriores conferencias, que hacían llamados para incrementar el uso de los medios en la evangelización, en Aparecida el Episcopado reconoce que la Iglesia latinoamericana cuenta ahora con un gran número de medios: "Tenemos radio, televisión, cine, prensa, internet, páginas web y la Red Informática de la Iglesia en América Latina, RIIAL, que nos Ilena de esperanza" (Documento de Aparecida, 2007, p. 26).

En el marco de la globalización y la cultura, Aparecida reconoce la vitalidad de la Iglesia en América latina y alaba este continente como espacio de encuentro de pueblos y culturas. Motiva a la pastoral a conocer y valorar la nueva cultura de la comunicación, promoviendo la formación profesional en ella. Puntualmente, Ilama 
a formar comunicadores profesionales competentes y comprometidos con los valores humanos en la transformación de la sociedad (Documento de Aparecida, 2007, p. 114). Igualmente, invita a formar a las personas en el uso adecuado de los medios de comunicación.

\section{CONCLUSIONES}

Se puede observar en los documentos de las cinco Conferencias del Episcopado Latinoamericano una preocupación explícita por los medios de comunicación y, por extensión, por la comunicación social.

Las preocupaciones de la iglesia toman diferentes direcciones de acuerdo con la coyuntura de las décadas de cada conferencia. Así, la primera se preocupa por usar la radio y la prensa, cuando no se había popularizado la televisión, y en la última hay preocupaciones por la globalización multimedial. En ese orden de ideas, el episcopado del continente reflexiona desde los avances mediáticos hasta su influencia en la sociedad de nuestros días.

Se pueden ver, consecuentemente, dos grandes aseveraciones: La iglesia latinoamericana reconoce las capacidades de los medios para difundir la palabra y, al mismo tiempo (sobre todo en las últimas conferencias) ve en ellos amenazas, sobre todo culturales y axiológicas, pues considera que estos propagan un cultura banal, que estimula la búsqueda de riqueza, amor y sexo libre y prematrimonial, entre otros. La Conferencia de Puebla es la que más explicita esa incidencia negativa de los medios, que fácilmente se puede ver como una lectura influenciada desde la teoría crítica.

Se destaca, de manera preponderante, la influencia de la Conferencia de Medellín, inmanente a la teología de la liberación, pues se observa un discurso crítico sobre las estructuras sociales de la dinámica capitalista. En esa línea, al hablar del cambio social, dice que los medios deben ser consecuentes con esta meta. En Medellín, el episcopado habla de la comunicación como una dimensión humana, más allá de su vinculación con los medios.

En general, en las conferencias se observa el interés por formar a sacerdotes y seminaristas en el campo de los medios. Si se hiciera esto desde la academia, se podría advertir una línea de comunicacióneducación. En una tendencia similar, en Santo Domingo se habla de conocer las características de los oyentes, es decir, caracterizar a los receptores y no verlos como una masa.

De manera llamativa, y respondiendo a preocupaciones de muchas comunidades del continente, la iglesia latinoamericana muestra en la última conferencia (2007) su preocupación por la amenaza que significa la globalización para las culturas locales, pues impone una cultura artificial. Esta preocupación, aún vigente en medios alternativos y comunitarios, mantiene la tensión global/local presente en la dinámica actual, desde los planos económicos, políticos, sociales y culturales.

\section{REFERENCIAS}

Babin, P. (2011). La era de la comunicación: Para un nuevo modo de evangelizar. Bilbao, España: Sal Terrae.

Documento de Aparecida. (2007). V Conferencia general del episcopado latinoamericano. Recuperado de http://www.caritas.org.ar/download/ Documento_Conclusivo_Aparecida.pdf. 
Documento de Medellín. (1968). II Conferencia general del episcopado latinoamericano. Recuperado de http:// www.caritas.org.ar/htm/iglesia-lato1. htm.

Documento de Puebla. (1979). III Conferencia general del episcopado latinoamericano. Recuperado de http:// www.caritas.org.ar/htm/iglesia-lat01. htm.

Documento de Río de Janeiro. (1955). I Conferencia general del episcopado latinoamericano. Recuperado de http:// www.caritas.org.ar/htm/iglesia-lato1. htm.

Documento de Santo Domingo. (1992). IV Conferencia general del episcopado latinoamericano. Recuperado de http:// www.caritas.org.ar/htm/iglesia-lato1. htm.

Pascual, J. (1976). Los medios de comunicación social en la doctrina de la iglesia. Madrid, España: Ministerio de Educación y Ciencia.

Raigón, G. (2010). Estructura de la información en la iglesia católica. Murcia, España: Universidad de Murcia.

Spoletini, B. (1977). Comunicación social e Iglesia: Documentos latinoamericanos 1959-1976. Bogotá, Colombia: Paulinas.

Spoletini, B. (1985). Comunicación e Iglesia Latinoamericana. Buenos Aires, Argentina: UNDA-AL. 